\title{
11 Superconductivity and Magnetism
}

D. Di Castro (since February 2002), H. Keller, R. Khasanov, S. Kohout (since September 2001), M. Mali, P. Morf (till Mai 2001),

R. Renggli (till Mai 2001), J. Roos, A. Schilling (till October 2001), Ph. Schneider (till Mai 2001), A. Shengelaya, G.M. Zhao (till June 2001),

V.A. Ivanshin (visiting scientist), E. Kirov (visiting scientist),

B. Kochelaev (visiting scientist), T. Schneider (Titularprofessor), and K.A. Müller (Honorarprofessor)

in collaboration with:

ETH Zürich (M. Angst, K. Conder, J. Karpinski), Paul Scherrer Institute (E. Morenzoni), IBM Rüschlikon Research Laboratory (J.G. Bednorz), University of Birmingham (E.M. Forgan), University of St. Andrews (S.L. Lee), University of Rome (A. Bianconi), Kazan State University (A. Dooglav, M.V. Eremin, B.I. Kochelaev), University of Belgrade (I.M. Savić), Institute of Low Temperature and Structure Research, Polish Academy of Sciences, Wroclaw (P.W. Klamut), Institute of Physics, Polish Academy of Sciences, Warsaw (A. Wisniewski), University of Tokyo (K. Kishio and T. Sasagawa), Northern Illinois University, DeKalb (B. Dabrowski), University of Maryland (R. L. Greene, T. Venkatesan, D. J. Kang, W. Prellier, M. Rajeswari), University of Berkeley (R.A. Fisher and N.E. Phillips), Los Alamos National Laboratory (M. Hundley, A. Lacerda), Argonne National Laboratory (U. Welp, W.K. Kwok, R.J. Olsson, G.W. Crabtree).

\section{$11.1 \quad$ Introduction}

In the last year we have continued our investigations on the fundamental physical properties of non-conventional (cuprates and $\mathrm{MgB}_{2}$ ) and conventional superconductors, manganites and other related perovskite systems. One central research topic involves detailed oxygen isotope $\left({ }^{16} \mathrm{O} /{ }^{18} \mathrm{O}\right)$ effect (OIE) studies on various physical quantities (critical temperature, in-plane penetration depth, resistivity, thermoelectric power, specific heat) in manganites and cuprates in order to investigate the role of the electron-phonon interaction in the basic physics of these systems. In another project, we have continued our studies of vortex matter in strongly layered superconductors (static vortex structure and vortex motion), that we have started several years ago. A great advantage of our approach is the combined application of complementary experimental techniques, such as muon-spin rotation ( $\mu \mathrm{SR})$, nuclear magnetic resonance (NMR), nuclear quadrupole resonance (NQR), electron paramagnetic resonance (EPR), together with bulk SQUID and torque magnetometry, resistivity, and thermal measurements. The scientific goal of our research is to gain new insight on the fundamental physical processes involved in cuprate superconductors and related systems.

\subsection{Studies of oxygen isotope effects}

\subsubsection{Oxygen isotope effect in manganites}

Although a consensus is reached that strong electron-phonon coupling plays an essential role in the basic physics of the colossal magnetoresistive manganites, the very nature of charge carriers in the ferromagnetic states at low temperatures has not been understood. One theory [1] shows that the nature of charge carriers is polaronic even in the low-temperature metallic state, while others $[2,3]$ believe that polaronic effects are not important.

In order to provide clear evidence for the 'metallic' polaronic liquid in the low temperature ferromagnetic state, it is essential to study the isotope effects on the low-temperature kinetic 
and thermodynamic properties. These properties are dominated by the impurity and electronelectron scatterings, so the only source of the isotope effect might be the polaron mass dependence on the lattice ion mass. For example, replacing ${ }^{16} \mathrm{O}$ with ${ }^{18} \mathrm{O}$ will increase the polaron mass, leading to the changes in the residual resistivity, and in other kinetic and thermodynamic properties.

We thus studied the isotope effects on the residual resistivity $\rho_{o}$, the thermoelectric power $S$ and the electronic specific heat $C_{e l}$ both experimentally and theoretically [4]. In the low-temperature ferromagnetic state, the intrinsic resistivity of the manganite thin films shows a strong dependence on the oxygen isotope mass. The residual resistivity of the films increases by $15(3) \%$ upon replacing ${ }^{16} \mathrm{O}$ with ${ }^{18} \mathrm{O}$. In contrast, the thermoelectric power is nearly independent of the oxygen isotope mass. The observed large isotope effect on the resistivity and negligible effect on the thermoelectric power are in quantitative agreement with our theory based on a novel polaronic Fermi liquid [4]. Furthermore, we have also found that $C_{e l}$ does not have a significant isotope dependence [5], in agreement with theory [4]. Therefore, our theoretical and experimental studies of the isotope effects on the kinetic and thermodynamic properties provide strong evidence for the polaronic Fermi-liquid in the low-temperature ferromagnetic state of doped manganites.

\subsubsection{Oxygen isotope effects in cuprates}

\section{a) Oxygen isotope effect on the magnetic penetration depth in underdoped $\mathbf{Y}_{1-x} \mathbf{P r}_{x} \mathbf{B a}_{2} \mathbf{C u}_{3} \mathbf{O}_{7-\delta}$}

There is increasing evidence that a strong electron-phonon coupling is present in cuprate superconductors, which may lead to the formation of polarons (bare charge carriers accompanied by local lattice distortions) [6,7]. One way to test this hypothesis is to demonstrate that the effective mass of the supercarriers $m^{*}$ depends on the mass $M$ of the lattice atoms. This is in contrast to conventional BCS superconductors, where $m^{*}$ is independent of $M$. For cuprate superconductors (clean limit) the in-plane penetration depth $\lambda_{a b}$ is given by $\lambda_{a b}^{-2}(0) \propto n_{s} / m_{a b}^{*}$, where $n_{s}$ is the superconducting charge carrier density, and $m_{a b}^{*}$ is the in-plane effective mass of the superconducting charge carriers. This implies that the OIE on $\lambda_{a b}^{-2}(0)$ is due to a shift in $n_{s}$ and/or $m_{a b}^{*}$, according to the relation:

$$
\Delta \lambda_{a b}^{-2}(0) / \lambda_{a b}^{-2}(0)=\Delta n_{s} / n_{s}-\Delta m_{a b}^{*} / m_{a b}^{*} .
$$

Therefore a possible mass dependence of $m_{a b}^{*}$ can be tested by investigating the isotope effect on $\lambda_{a b}$, provided that the contribution of $n_{s}$ to the total isotope shift is known.

The muon-spin rotation ( $\mu \mathrm{SR})$ technique is a powerful method to determine directly the magnetic penetration depth in type-II superconductors. In a powder sample the magnetic penetration depth $\lambda$ can be extracted from the superconducting part of the muon-spin depolarisation rate $\sigma_{s c}(T) \propto 1 / \lambda^{2}(T)$, which probes the second moment $\left\langle\Delta B^{2}\right\rangle^{1 / 2}$ of the local magnetic field distribution $p(B)$ in the mixed state [8]. For the highly anisotropic layered cuprate superconductors the simple relation $\sigma_{s c}(T) \propto 1 / \lambda_{a b}^{2}(T) \propto n_{s} / m_{a b}^{*}$ holds [8].

We performed a detailed investigation of the OIE on the in-plane penetration depth $\lambda_{a b}(0)$ in underdoped $\mathrm{Y}_{1-x} \mathrm{Pr}_{x} \mathrm{Ba}_{2} \mathrm{Cu}_{3} \mathrm{O}_{7-\delta}(x=0.3$ and 0.4) by means of $\mu$ SR [9]. For both concentrations $x$ a pronounced OIE on $T_{c}$ as well on $\lambda_{a b}^{-2}(0)$ was observed. The corresponding results for $x=0.4$ are displayed in Fig. 11.1. The relative isotope shifts on $\lambda_{a b}^{-2}(0)$ were found to be $\Delta \lambda_{a b}^{-2}(0) / \lambda_{a b}^{-2}(0)=-5(2) \%$ for $x=0.3$ and $-9(2) \%$ for $x=0.4$. These results are in excellent agreement with recent results of our group obtained for underdoped $\mathrm{La}_{2-x} \mathrm{Sr}_{x} \mathrm{CuO}_{4}$ by means of torque magnetometry [10]. According to Eq (11.1) the observed $\Delta \lambda_{a b}^{-2}(0) / \lambda_{a b}^{-2}(0)$ is due to a shift of $n_{s}$ and/or $m_{a b}^{*}$. For $\mathrm{La}_{2-x} \mathrm{Sr}_{x} \mathrm{CuO}_{4}$ several independent experiments 

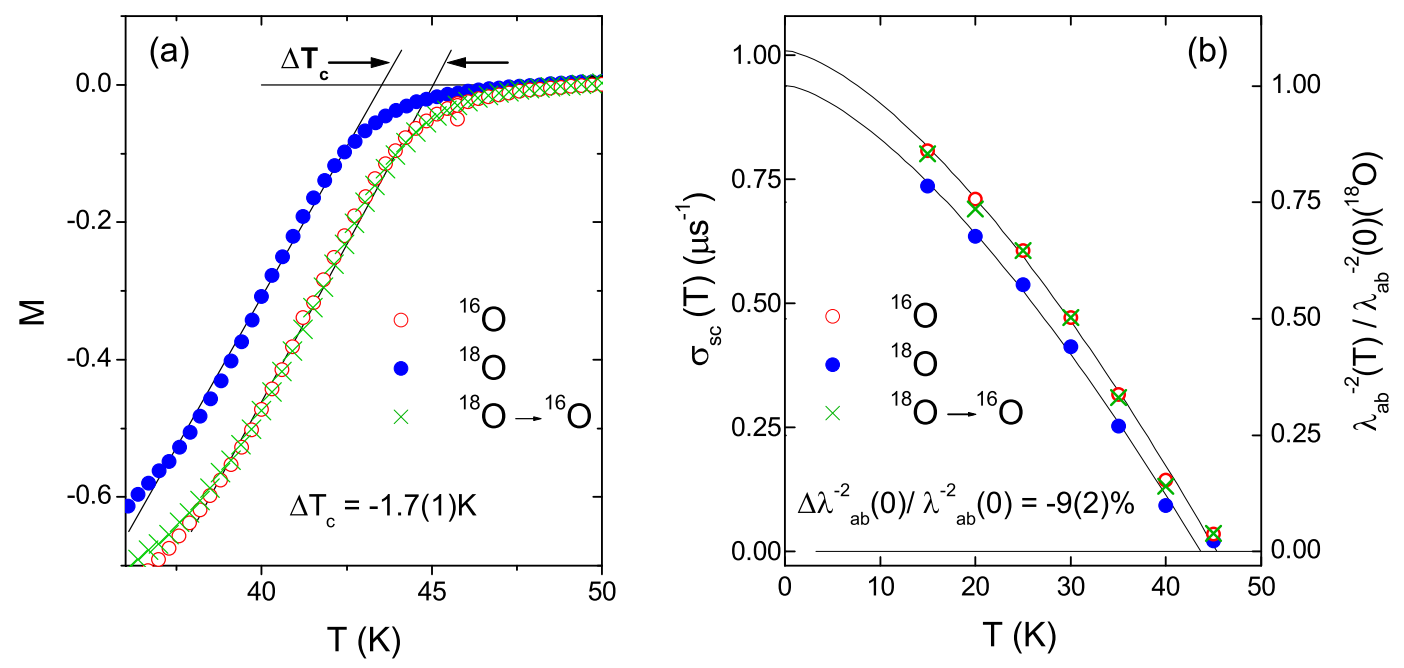

Figure 11.1: (a) Low-field (1 mT, field-cooled) magnetisation curves (normalised at 10K) near $T_{c}$ for $Y_{0.6} \operatorname{Pr}_{0.4} \mathrm{Ba}_{2} \mathrm{Cu}_{3} \mathrm{O}_{7-\delta}$. (b) Temperature dependence of the depolarization rate $\sigma_{s c}$ of $Y_{0.6} \mathrm{Pr}_{0.4} \mathrm{Ba}_{2} \mathrm{Cu}_{3} \mathrm{O}_{7-\delta}$ measured in a field of $200 \mathrm{mT}$ after field-cooling the sample from the normal state. The solid lines correspond to fits to the power law: $\sigma_{s c}(T) / \sigma_{s c}(0)=1-\left(T / T_{c}\right)^{n}$.

$[10,11,12]$ have shown that the change of $n_{s}$ during the exchange procedure must be negligibly small. The same arguments also apply for the $\mathrm{Y}_{1-x} \mathrm{Pr}_{x} \mathrm{Ba}_{2} \mathrm{Cu}_{3} \mathrm{O}_{7-\delta}$ system [9]. This implies that the observed change in $\lambda_{a b}^{-2}(0)$ is mainly due to the OIE on the in-plane effective mass $m_{a b}^{*}$ with $\Delta m_{a b}^{*} / m_{a b}^{*} \simeq 5(2) \%$ and $9(2) \%$ for $x=0.3$ and $x=0.4$, respectively.

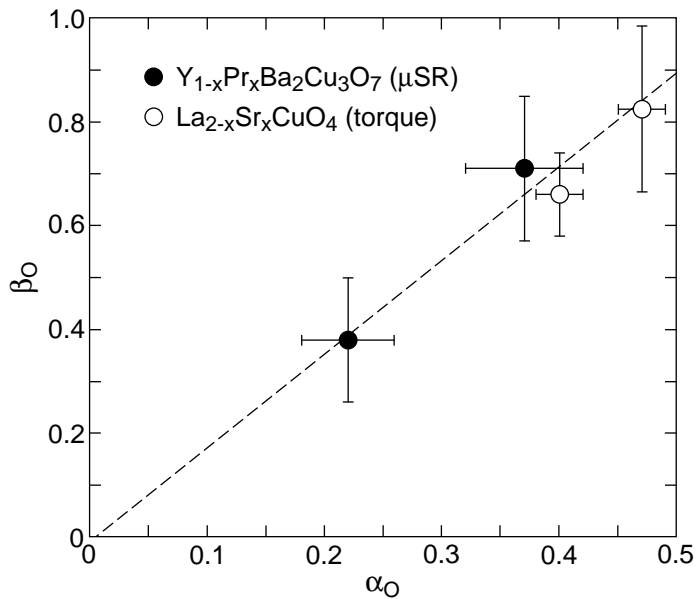

Figure 11.2: Plot of the OIE exponent $\beta_{\mathrm{O}}$ versus the OIE exponent $\alpha_{\mathrm{O}}$ for $\mathrm{Y}_{1-x} \mathrm{Pr}_{x} \mathrm{Ba}_{2} \mathrm{Cu}_{3} \mathrm{O}_{7-\delta}$ $(x=0.3$ and 0.4$)$ and $\mathrm{La}_{2-x} \mathrm{Sr}_{x} \mathrm{CuO}_{4}(x=0.080$ and 0.086) from [10]. The dashed line represents a best fit to the empirical relation $\beta_{\mathrm{O}}=A \cdot \alpha_{\mathrm{O}}+B$ with $A=1.8(4)$ and $B=-0.01(12)$.

In Fig. 11.2 the OIE exponent $\beta_{\mathrm{O}}$ of $\lambda_{a b}^{-2}(0)$ is plotted versus the OIE exponent $\alpha_{\mathrm{O}}$ of $T_{c}$ for $\mathrm{Y}_{1-x} \mathrm{Pr}_{x} \mathrm{Ba}_{2} \mathrm{Cu}_{3} \mathrm{O}_{7-\delta}$, where $\beta_{\mathrm{O}}=-d \ln \lambda_{a b}^{-2}(0) / d \ln M_{\mathrm{O}}$ and $\alpha_{\mathrm{O}}=-d \ln T_{c} / d \ln M_{\mathrm{O}}$. For comparison the recent magnetic torque results of underdoped $\mathrm{La}_{2-x} \mathrm{Sr}_{x} \mathrm{CuO}_{4}$ [10] are also included. Evidently $\beta_{\mathrm{O}}$ and $\alpha_{\mathrm{O}}$ are proportional to each other with $\beta_{\mathrm{O}} / \alpha_{\mathrm{O}} \simeq 1.8(4)$. This empirical relation appears to be generic for various families of cuprate superconductors.

In conclusion, our OIE studies imply that the effective supercarrier mass $m_{a b}^{*}$ in underdoped $\mathrm{Y}_{1-x} \mathrm{Pr}_{x} \mathrm{Ba}_{2} \mathrm{Cu}_{3} \mathrm{O}_{7-\delta}$ depends on the oxygen mass of the lattice atoms, which is not expected for a conventional phonon-mediated BCS superconductor. To our knowledge there are only two theoretical models of high-temperature superconductivity, which predict an OIE on the effective carrier mass, namely, a bipolaronic model of Alexandrov and Mott [6] and a nonadiabatic superconductivity model proposed by Grimaldi, Cappelluti and Pietronero [13]. 


\section{b) Low-energy $\mu \mathrm{SR}$ investigation of the oxygen isotope effect on the magnetic penetration depth of $\mathrm{YBa}_{2} \mathrm{Cu}_{3} \mathrm{O}_{7-\delta}$}

At present the novel low-energy (LE) $\mu$ SR technique recently developed at PSI [14] is the most direct method to determine the magnetic penetration depth $\lambda$ in a superconductor. It allows to measure directly the magnetic field profile beneath the surface of a superconductor in the Meissner state, with a depth resolution of a few nanometers [15]. It is well known, that for superconductors in the clean limit, a magnetic field $B_{0}$ applied parallel to the surface of a semiinfinite slab decays with depth $z$ according to the exponential law: $B(z)=B_{0} \exp \left(-z / \lambda_{a b}\right)$.

We performed LE $\mu \mathrm{SR}$ studies of the OIE on the in-plane magnetic penetration depth $\lambda_{a b}$ in a $c$-axis oriented $\mathrm{YBa}_{2} \mathrm{Cu}_{3} \mathrm{O}_{7-\delta}$ film in the Meissner state. During the experiments the magnetic field $B_{0}=9.5 \mathrm{mT}$ was applied parallel to the surface of the $600 \mathrm{~nm}$ thick $\mathrm{YBa}_{2} \mathrm{Cu}_{3} \mathrm{O}_{7-\delta}$ film after zero-field cooling the film to $5 \mathrm{~K}$ from the normal state. In this ge-
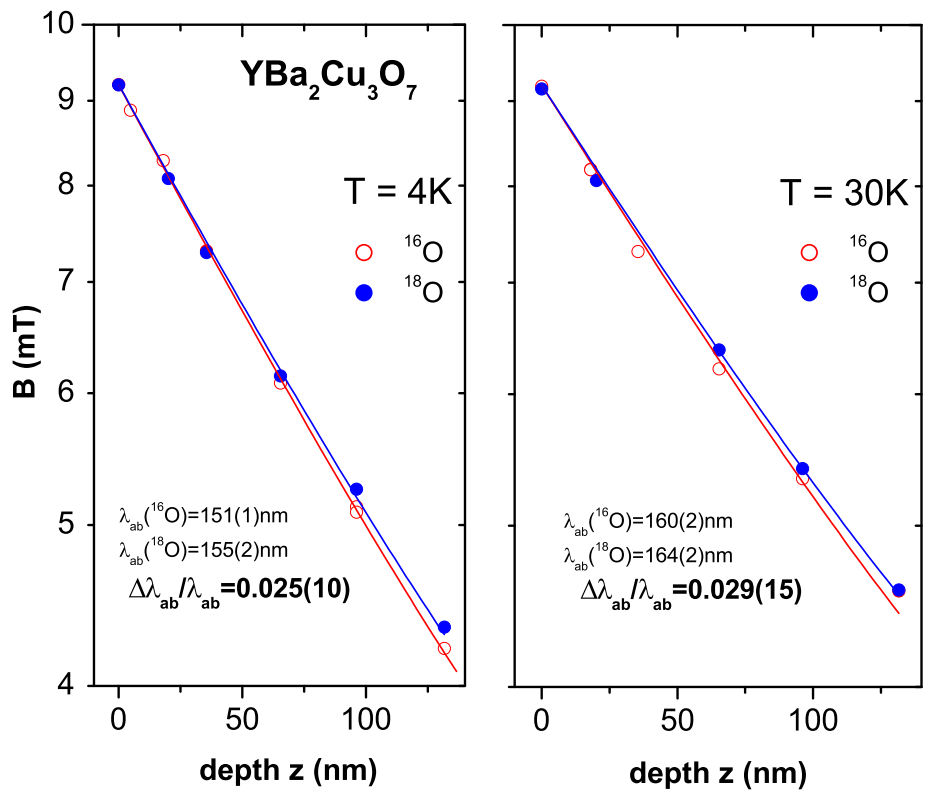

Figure 11.3: Magnetic field profile $B(z)$ in a $600 \mathrm{~nm}$ thick $\mathrm{c}$ axis oriented $\mathrm{YBa}_{2} \mathrm{Cu}_{3} \mathrm{O}_{7-\delta}$ film in the Meissner state near the surface for ${ }^{16} \mathrm{O}$ and ${ }^{18} \mathrm{O}$ at two different temperatures. Solid curves represent fits to the theoretical expression given in the text.

ometry, currents flowing in the $a b$-planes determine the magnetic field profile along the $c$-axis inside the film. LE muons were implanted at depths ranging from 40 to $150 \mathrm{~nm}$ by varying the energy of the incident muons from 5 to $30 \mathrm{keV}$. Fig. 11.3 shows the measured magnetic field profile in the Meissner state of the $\mathrm{YBa}_{2} \mathrm{Cu}_{3} \mathrm{O}_{7-\delta}$ thin film for ${ }^{16} \mathrm{O}$ and ${ }^{18} \mathrm{O}$ samples. It is evident that the magnetic field inside the film decays stronger for the ${ }^{16} \mathrm{O}$ sample than for the ${ }^{18} \mathrm{O}$ one, implying that $\lambda_{a b}$ is smaller for ${ }^{16} \mathrm{O}$ than for ${ }^{18} \mathrm{O}$. The relative oxygen-isotope shifts extracted from these preliminary data were found to be $\Delta \lambda_{a b} / \lambda_{a b}=0.025(10)$ and $0.029(15)$ at $T=4 \mathrm{~K}$ and $30 \mathrm{~K}$, respectively. This result clearly indicates that lattice effects play an essential role also in optimally doped cuprate superconductors, in contrast to the general belief. These effects have to be considered in any successful theory of high-temperature superconductivity.

\section{c) Antiferromagnetic transition}

It is well known that the parent compounds of the cuprate superconductors exhibit long-range $3 \mathrm{D}$ antiferromagnetic (AF) order, which is rapidly destroyed as holes are doped into the $\mathrm{CuO}_{2}$ planes. However, short-range 2D AF correlations survive well into the superconducting region of the phase diagram. There is also increasing evidence that a strong electron-phonon coupling 
is present in cuprates [16] which may lead to the formation of polarons (bare charge carriers accompanied by local lattice distortions) [6]. On the other hand, little is known about the influence of the electron-phonon interaction on magnetism in cuprates. Conventional theories of magnetism neglect atomic vibrations; the atoms are generally considered as infinitely heavy and static in theoretical descriptions of magnetic phenomena, so there should be no isotope effect on magnetism. However, if charge carriers are polaronic, i.e., nuclear and electronic motions are no longer decoupled (breakdown of the Born-Oppenheimer approximation), one might expect isotope effects on magnetic properties. The question is whether such an isotope effect exists in the cuprates.

To answer this question we previously studied the OIE on low-temperature magnetism in $\mathrm{La}_{2-x} \mathrm{Sr}_{x} \mathrm{CuO}_{4}(x=0.03,0.05)$ using the zero-field $\mu \mathrm{SR}$ technique [17]. In these samples a so-called cluster spin glass magnetic state is present below the spin glass freezing temperature $T_{g}$. We observed that $T_{g}$ is isotope dependent: $T_{g}=8.60(2) \mathrm{K}$ and $8.80(2) \mathrm{K}$ for the ${ }^{16} \mathrm{O}$ and ${ }^{18} \mathrm{O}$ sample, respectively. This results in a OIE exponent for the spin glass freezing temperature $\alpha_{T_{g}}=-d \ln T_{g} / d \ln M=-0.19(1)$. It is important to show that this novel isotope effect is generic and also exists in other cuprate families.

This time we have studied the OIE on magnetism in $\mathrm{Y}_{0.2} \mathrm{Pr}_{0.8} \mathrm{Ba}_{2} \mathrm{Cu}_{3} \mathrm{O}_{7-\delta}$. It is known that $\mathrm{Pr}$ doping in $\mathrm{YBa}_{2} \mathrm{Cu}_{3} \mathrm{O}_{7-\delta}$ suppresses superconductivity, and long-range antiferromagnetic order is induced [18]. The $\mu \mathrm{SR}$ experiments were performed at the Paul Scherrer Institute in Villigen, Switzerland, using essentially $100 \%$ spin-polarized positive "surface muons". The pair of samples with different oxygen isotopes ${ }^{16} \mathrm{O}$ and ${ }^{18} \mathrm{O}$ were measured under the same experimental conditions.

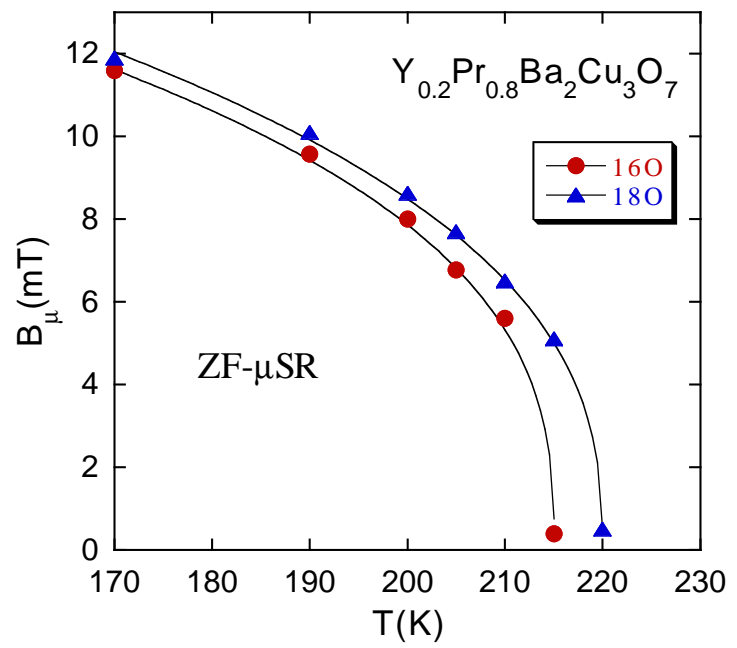

Figure 11.4: Temperature dependence of the internal magnetic field $B_{\mu}$ at the muon site for the ${ }^{16} \mathrm{O}$ and ${ }^{18} \mathrm{O}$ samples of $Y_{0.2} \mathrm{Pr}_{0.8} \mathrm{Ba}_{2} \mathrm{Cu}_{3} \mathrm{O}_{7-\delta}$. Solid lines represent fitted curves to the power law $B_{\mu}(T)=$ $B_{\mu}(0)\left(1-T / T_{N}\right)^{n}$.

At low temperatures in all studied samples we observed damped oscillations due to muonspin precession in local magnetic fields. A clear oscillation observed in ZF- $\mu$ SR spectra implies that the muons sense a well defined internal magnetic field, in agreement with previous $\mu \mathrm{SR}$ measurements [18]. Fig. 11.4 shows the internal magnetic field $B_{\mu}$ for the two oxygen isotope $\left({ }^{16} \mathrm{O}\right.$ and $\left.{ }^{18} \mathrm{O}\right)$ samples as a function of temperature. One can see that the antiferromagnetic transition temperature $T_{N}$ is isotope dependent. $T_{N}$ of the ${ }^{18} \mathrm{O}$ sample is $5 \mathrm{~K}$ higher than that of the ${ }^{16} \mathrm{O}$ sample, yielding an oxygen isotope exponent $\alpha_{T_{N}}=-d \ln T_{N} / d \ln M=-0.19(1)$. It is remarkable that the values of the isotope exponents $\alpha_{T_{g}}$ in $\mathrm{La}_{2-x} \mathrm{Sr}_{x} \mathrm{CuO}_{4}$ and $\alpha_{T_{N}}$ in $\mathrm{Y}_{0.2} \mathrm{Pr}_{0.8} \mathrm{Ba}_{2} \mathrm{Cu}_{3} \mathrm{O}_{7-\delta}$ are the same. Moreover, our results imply that the isotope effect on magnetism is a generic phenomenon in cuprate systems. This novel isotope effect clearly demonstrates that the lattice vibrations strongly affect magnetism in cuprates which may be explained by taking into account the polaronic nature of the charge carriers. 


\subsection{Studies of $\mathrm{MgB}_{2}$}

The recent discovery of superconductivity in $\mathrm{MgB}_{2}$ by Nagamatsu et al. [19] has lead in our group to a number of investigations with the aim to understand the intrinsic properties of this structurally layered and thus highly anisotropic compound. Its transition temperature of $39 \mathrm{~K}$ is on the limit expected theoretically for BCS, phonon mediated superconductivity, and its superconducting properties are rather unusual.

\subsubsection{NMR investigations}

So far, NMR studies of $\mathrm{MgB}_{2}$ were performed by using the ${ }^{11} \mathrm{~B}$ nuclei as microscopic probes and there are no experimental data concerning the NMR quantities at the $\mathrm{Mg}$ site in $\mathrm{MgB}_{2}$, although theoretical predictions based on ab initio local density approximation (LDA) calculations exist for $\mathrm{Mg}$ Knight shift, spin-lattice relaxation, $1 / T_{1}$, and electric field gradient (EFG) $[20,21]$.

The only NMR active ${ }^{25} \mathrm{Mg}$ isotope has a small magnetic moment and low natural abundance and consequently the ${ }^{25} \mathrm{Mg}$ NMR signals are weak even in high magnetic fields. Despite this handicap we were able to investigate NMR of the naturally abundant ${ }^{25} \mathrm{Mg}$ in powder samples of $\mathrm{MgB}_{2}$. We measured the temperature dependence of Knight shift, spin-lattice relaxation time, and quadrupole coupling frequency of ${ }^{25} \mathrm{Mg}$ in $\mathrm{MgB}_{2}$ [22]. Figure 11.5 shows a ${ }^{25} \mathrm{Mg}$ powder NMR spectrum at room temperature and the temperature independent Knight shift, $K_{c}$, in normal conducting $\mathrm{MgB}_{2}$ powder grains with the c axis parallel to the applied magnetic field. From our results for the linearly temperature dependent spin-lattice relax-
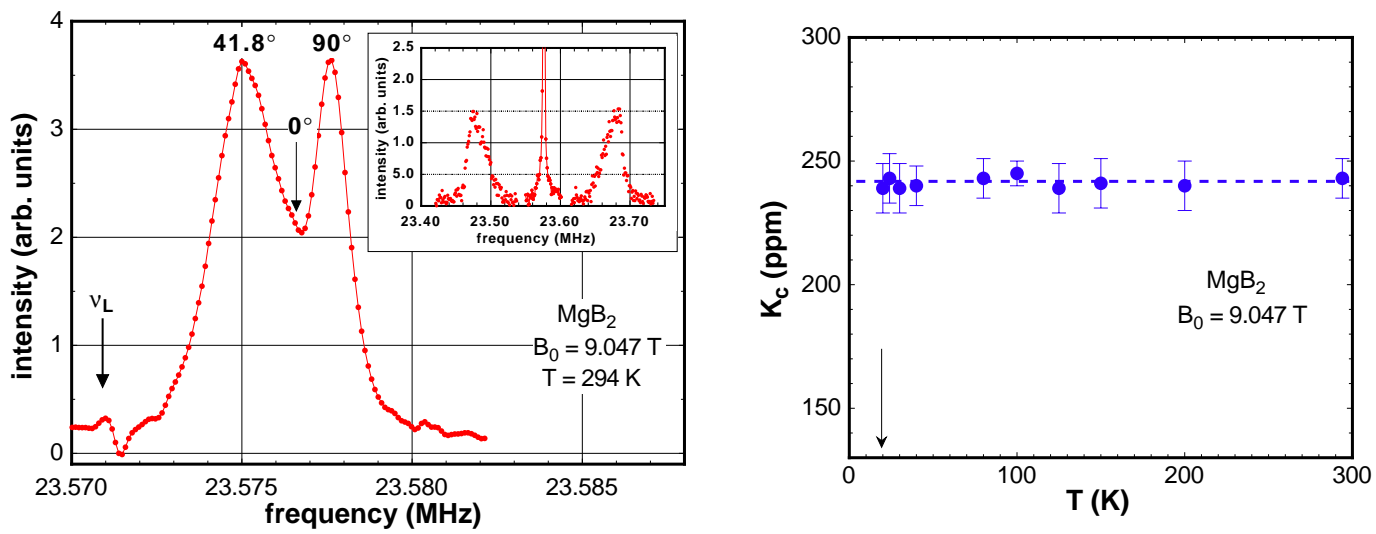

Figure 11.5: Left: ${ }^{25} \mathrm{Mg}$ central line of $\mathrm{MgB}_{2}$ powder spectrum measured at $T=294 \mathrm{~K}$ in a magnetic field $B_{0}=9.047 \mathrm{~T}$. Insert: ${ }^{25} \mathrm{Mg}$ first satellites' singularities of $\mathrm{MgB}_{2}$ powder spectrum measured at $T=294 \mathrm{~K}$ in a magnetic field $B_{0}=9.047 \mathrm{~T}$. Right: Temperature dependence of the ${ }^{25} \mathrm{Mg}$ Knight shift, $K_{c}$, in normal conducting $\mathrm{MgB}_{2}$ powder grains with $c$ $\| B_{0}$. The shift is with respect to the ${ }^{25} \mathrm{Mg}$ resonance in a water solution of $\mathrm{MgCl}_{2}$ at room temperature. The dashed line represents the weighted average of $K_{c}$. The arrow marks the transition temperature of $\mathrm{MgB}_{2}$ powder grains with $c \perp B_{0}=9.047 \mathrm{~T}$.

ation time, $T_{1}$, the Korringa ratio can be formed. We get experimentally $\left(K^{2} T_{1} T\right) / S \approx 0.95$, where $S=\left(\gamma_{e} / \gamma_{n}\right)^{2}\left(h / 8 \pi^{2} k_{B}\right)=7.0323 \times 10^{-5}$ sK for ${ }^{25} \mathrm{Mg}$, with $\gamma_{e}$ and $\gamma_{n}$ the gyromagnetic ratios for electron and nucleus, respectively. This value is very close to the ideal value of unity for s-electrons. This result is a confirmation that the Fermi-contact interaction is the dominant mechanism responsible for relaxation and Knight shift at the Mg site. 
Comparing our experimental results with the theoretical predictions [20, 21], we find for all three Mg NMR quantities an excellent agreement. Since all calculations were done by state of the art LDA methods the good agreement between calculated and measured NMR quantities confirms the LDA as a good approximation for $\mathrm{MgB}_{2}$.

\subsubsection{Micro-torque measurements}

In a collaboration with M. Angst and J. Karpinski (Laboratorium für Festkörperphysik, ETH Zürich) high quality single crystals of $\mathrm{MgB}_{2}\left(T_{c} \approx 38-39 \mathrm{~K}, \Delta T_{c} \leq 0.5 \mathrm{~K}\right)$ were used in an investigation of the upper critical field of this novel superconductor with torque magnetometry [23]. As shown in Fig. $11.6 H_{c 2}^{\| c}$ is very low $(2.5 \mathrm{~T}$ at the temperature of
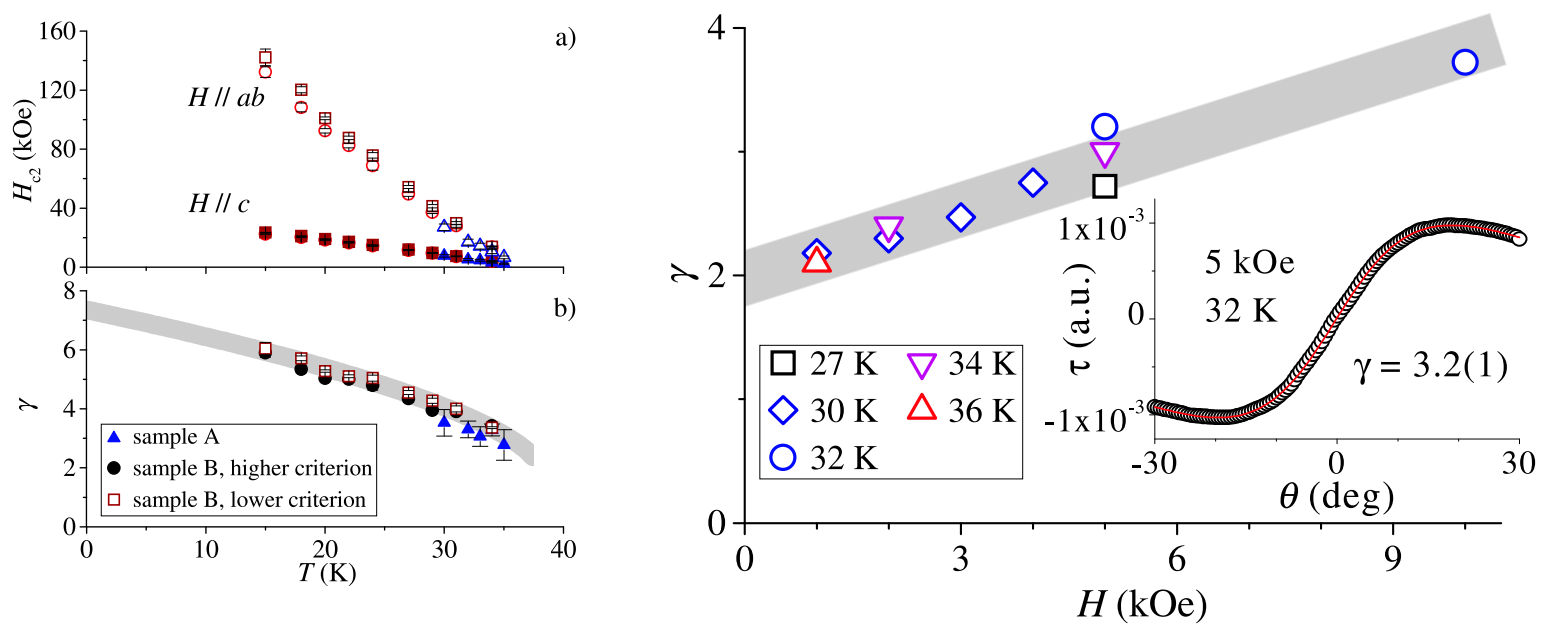

Figure 11.6: Left side: Upper critical field $H_{\mathrm{c} 2}(a)$ and $H_{\mathrm{c} 2}$ anisotropy $\gamma(b)$ vs temperature. Right side: Anisotropy $\gamma$ as a function of field, inset shows the fitting of torque vs angle data.

$15 \mathrm{~K}$ ) and saturates with decreasing temperature, while $H_{c 2}^{\| a b}$ increases at low temperatures up to about $14 \mathrm{~T}$ at $15 \mathrm{~K}$. The upper critical field anisotropy $\gamma$ was found to be temperature dependent (decreasing from $\gamma \simeq 6$ at $15 \mathrm{~K}$ to 2.8 at $35 \mathrm{~K}$ ). The anisotropy deduced from reversible torque data near $T_{\mathrm{c}}$ is also field dependent (increasing roughly linearly from $\gamma \simeq 2$ in zero field to 3.7 in $10 \mathrm{kOe}$ ). Such results imply a breakdown of standard anisotropic Ginzburg-Landau theory with a temperature and field independent effective mass anisotropy. A quantitative explanation of the measured $\gamma(T)$ has to take into account both the wavevector dependence of the band effective mass tensor and the anisotropic electron-phonon coupling. The latter can lead to an anisotropic energy gap (or two gaps), which can result in a temperature dependent anisotropy of the upper critical field.

\subsection{Vortex-matter studies}

\subsubsection{Possible phase transitions in the mixed state of $2 \mathrm{H}-\mathrm{NbSe}_{2}$ and $\mathrm{LuNi}_{2} \mathrm{~B}_{2} \mathrm{C}$}

Various experiments on the type-II superconductor $2 \mathrm{H}-\mathrm{NbSe}_{2}$ have suggested that a phase transition from an ordered to a disordered vortex phase below the upper critical field $H_{c 2}$ may take place [24]. However, no calorimetric evidence for such a phase transition has been reported so far. Corresponding features in heat capacity are indeed expected to be extremely small when compared to the total heat capacity of the material. We have therefore done a 
series of 140 high-resolution heat-capacity measurements on a $19 \mathrm{mg} 2 \mathrm{H}-\mathrm{NbSe}_{2}$ single crystal with a critical temperature $T_{c}=7.2 \mathrm{~K}$, that was also characterised by detailed magnetisation measurements (in collaboration with G. Ravikumar, Forschungszentrum Karlsruhe). In all these experiments, we have not found any features in the heat capacity that would support the scenario of a vortex-related thermodynamic phase transition. However, since the transition is expected to occur in the magnetically irreversible region of the $H$ - $T$ diagram, we may expect metastable vortex states, and thermodynamic equilibrium may be difficult to achieve [24].

A rich magnetic phase diagram has been discovered by neutron-scattering experiments in the borocarbide compound $\mathrm{LuNi}_{2} \mathrm{~B}_{2} \mathrm{C}$, where a transition from a cubic to a hexagonal vortex phase takes place [25]. This transition is most likely of second order [26]. We have studied a $\mathrm{LuNi}_{2} \mathrm{~B}_{2} \mathrm{C}$ single crystal with $T_{c}=16.6 \mathrm{~K}$ (in collaboration with P. Canfield and V. Kogan, Iowa State University) that was taken from the same batch as the samples that have been used for the neutron-scattering experiments. Again, no significant discontinuities suggesting a thermodynamic phase transition within the vortex state could be detected. As in the case of $2 \mathrm{H}-\mathrm{NbSe}_{2}$, the corresponding solid vortex phases are subject to pinning and irreversibility, which may make a measurement in thermodynamic equilibrium impossible.

We have therefore adapted a technique, that has been developed earlier at the University of Zürich, to accelerate thermodynamic equilibrium in the vortex state by applying an external transverse ac field $[27,28]$. The corresponding heat-capacity cell has been constructed and tested, but has not yet been used to continue the investigations on $2 \mathrm{H}-\mathrm{NbSe}_{2}$ and $\mathrm{LuNi}_{2} \mathrm{~B}_{2} \mathrm{C}$.

\subsection{2 ${ }^{63} \mathrm{Cu}$ NMR linewidth in $\mathrm{YBa}_{2} \mathrm{Cu}_{3} \mathrm{O}_{7}$}

We have studied in detail thermodynamic quantities (magnetisation and heat capacity) in connection with the first-order melting of the vortex lattice in $\mathrm{YBa}_{2} \mathrm{Cu}_{3} \mathrm{O}_{7}[29,30,31,32]$. However, these investigations did not allow us to draw any conclusions about microscopic properties of the vortex arrangement. It is known from neutron-scattering experiments [33] that above the first-order transition (i.e., in the vortex fluid state), no static spatial order exists on a time scale of the order of seconds. Moreover, muon-spin rotation experiments on $\mathrm{Bi}_{2} \mathrm{Sr}_{2} \mathrm{CaCu}_{2} \mathrm{O}_{8}$ have shown that no hexagonal order is present in the respective fluid phase on a time scale of microseconds [34].

NMR experiments would be, in principle, of great advantage to study the local magneticfield distribution in the mixed state of type-II superconductors because the method is relatively simple and inexpensive compared to other techniques. Early measurements of the ${ }^{89} \mathrm{Y}$ NMR linewidth on $\mathrm{YBa}_{2} \mathrm{Cu}_{4} \mathrm{O}_{8}$ powders, however, have not revealed any distinct signatures that could be clearly related to a phase transition between vortex phases [35]. We have therefore performed a linewidth measurement of the plane ${ }^{63} \mathrm{Cu} \mathrm{NMR}$ central line on a $\mathrm{YBa}_{2} \mathrm{Cu}_{3} \mathrm{O}_{7}$ single crystal that has been previously characterised by magnetisation and specific-heat measurements [29]. The NMR linewidth experiment is sensitive to spatial magnetic-field distributions that vary on a time scale of $\mu$ s and longer, and thus can serve as a tool to distinguish between static and dynamic vortex phases.

The NMR measurements have been done on an untwinned $\mathrm{YBa}_{2} \mathrm{Cu}_{3} \mathrm{O}_{7}$ single crystal in an external magnetic field $\mu_{0} H=9 \mathrm{~T}$ parallel to the $c$-axis. The linewidth $\Delta \nu_{1 / 2}$ decreases monotonically with increasing temperature $T$ in the vortex solid phase, but dramatically drops by $15 \%$ within $3 \mathrm{~K}$ at $T_{m} \approx 77 \mathrm{~K}$ (see Fig. 11.7), the melting temperature of the vortex lattice as determined by calorimetric measurements. This motional narrowing-effect is a clear indication for a transition from a static, ordered vortex phase to a fluid-like phase with no static order on the time scale of the measurement. It is interesting to note that there is a further small but discontinuous decrease in $\Delta \nu_{1 / 2}$ by $2 \%$ within the fluid phase 
(i.e., around $T=81 \mathrm{~K}$ ), that has no counterpart in our specific-heat data. Above $83 \mathrm{~K}$, and particularly at $T_{c}\left(\mu_{0} H=9 \mathrm{~T}\right) \approx 86 \mathrm{~K}$, no further anomalies in $\Delta \nu_{1 / 2}$ could be detected.

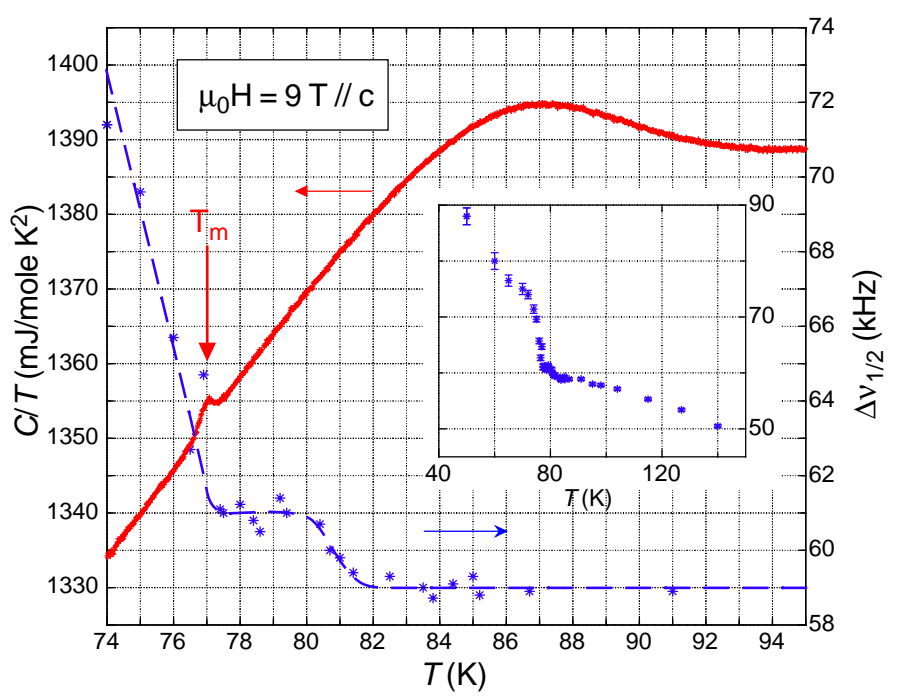

Figure 11.7: ${ }^{63} \mathrm{Cu}$ NMR half linewidth $\Delta \nu_{1 / 2}$ and reduced specific heat $\mathrm{C} / \mathrm{T}$ of $\mathrm{YBa}_{2} \mathrm{Cu}_{3} \mathrm{O}_{7}$ in $\mu_{0} \mathrm{H}=$ $9 \mathrm{~T}$ parallel to the c-axis. $\Delta \nu_{1 / 2}$ drops by $15 \%$ at the vortex-lattice melting temperature $T_{m} \approx 77 \mathrm{~K}$, where $C / T$ shows a sharp firstorder like peak.

The NMR investigations suggest that the local magnetic-field distribution changes discontinuously even within the fluid vortex phase of $\mathrm{YBa}_{2} \mathrm{Cu}_{3} \mathrm{O}_{7}$, indicating the presence of several possible fluid vortex phases. Although we could not confirm a corresponding phase transition between such phases by specific-heat measurements in $\mu_{0} H=9 \mathrm{~T}$, there is calorimetric evidence that beyond the upper critical point (i.e., above $\mu_{0} H=12 \mathrm{~T}$ where the liquefaction of the vortex lattice is continuous), several fluid vortex phases may exist [36] (collaboration with UC Berkeley, U.S.A., and Argonne National Laboratory, U.S.A.). The corresponding phase boundary is deep in the magnetically reversible region and well above the temperatures, where the solid-to-fluid transition is expected to take place. Both the magnitude and the sign of the observed discontinuities in specific heat $\left(\Delta C / T \approx+1 \mathrm{~mJ} / \mathrm{MolK}^{2}\right)$ raise puzzling questions concerning the symmetry of the corresponding phases [36] and the number of thermodynamic degrees of freedom involved in the phase transition [37].

\subsubsection{Vortex motion in type-II superconductors probed by $\mu \mathrm{SR}$}

It is well known that when a transport current is flowing in a type-II superconductor in the mixed state, the vortex lattice may be depinned from the pinning centers and may move freely due to the Lorentz force [38]. The $\mu \mathrm{SR}$ technique is an ideal tool to investigate vortex motion on a microscopic scale by investigating the local magnetic flux distribution $p(B)$ in the presence of a transport current. The first $\mu \mathrm{SR}$ experiment on vortex motion in a hightemperature superconductor was performed by our group several years ago on a ceramic sample of $\mathrm{YBa}_{2} \mathrm{Cu}_{3} \mathrm{O}_{7-\delta}$ [39].

Recently, we were able to observe vortex motion in a conventional type II superconductor by means of $\mu \mathrm{SR}$. The experiments were carried out at ISIS (Rutherford Appleton Laboratory, U.K.) which provides a pulsed muon beam with longitudinal polarisation. The sample was a polycrystalline $\mathrm{Pb}_{0.8} \mathrm{In}_{0.2}$ alloy with dimensions of $20 \times 30 \times 1 \mathrm{~mm}^{3}$. It was mounted at $45^{\circ}$ relative to the muon beam direction, and the magnetic field $(0.03 \mathrm{mT})$ was applied perpendicular to the incoming muon momentum. Transport currents (up to 80A) were applied along the largest dimension of the sample and at right angles to the magnetic field and the muon beam direction, giving rise to vortex motion (see Fig. 11.8).

Figure 11.9 shows a series of local magnetic field distributions $p(B)$ (determined from 


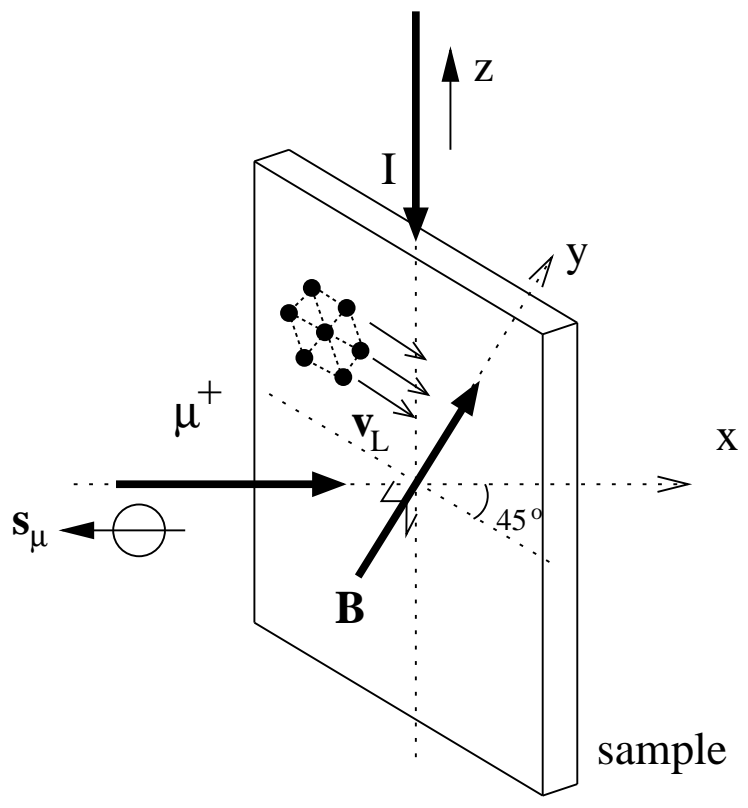

Figure 11.8: Schematic diagram of the experimental setup used for the $\mu S R$ experiments to detect vortex motion.

the $\mu \mathrm{SR}$ time spectra using a maximum entropy algorithm) for various transport currents, ranging from 0 to $80 \mathrm{~A}$. It can be seen that as the vortices move faster, $p(B)$ becomes narrower due to motional narrowing, i.e. muons experience an effective magnetic field closer to the average field. At the highest current (high vortex velocity) the magnetic field inhomogeneity in the mixed state is almost completely averaged out, and $p(B)$ tends to have the form of a delta function [40]. More detailed experiments are in progress.

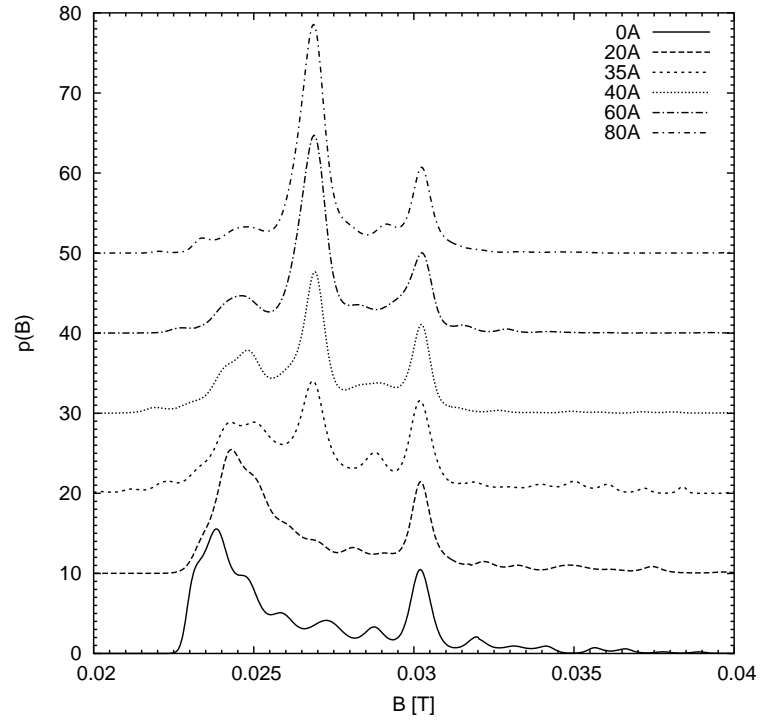

Figure 11.9: Vortex motion in a polycrystalline $P b_{0.8} I n_{0.2}$ alloy. The $\mu$ SR lineshapes $p(B)$ were obtained in a magnetic field of $0.03 \mathrm{mT}$ at $4.2 \mathrm{~K}$ for various applied currents, ranging from 0 to $80 \mathrm{~A}$. The peak at approximately $0.03 \mathrm{mT}$ arises from muons stopping in the sample holder and cryostat windows.

\subsection{Charge effects investigated by magnetic resonance techniques}

\subsubsection{EPR studies of $\mathrm{SrTiO}_{3}$}

Materials showing reversible resistive switching are attractive for today's semiconductor technology with its wide interest in nonvolatile random-access memories (RAM). Recently researchers at IBM Rüschlikon found very interesting phenomena of resistive switching with 
a memory effect in transition metal doped thin films of $\mathrm{SrTiO}_{3}$ [41]. They observed that positive or negative voltage pulses can induce resistivity changes of thin films up to six orders of magnitude. The switching time is shorter than $100 \mathrm{~ns}$. Once a low resistivity state has been achieved it persists without external power for months, demonstrating the feasibility of nonvolatile memory elements. Recently this effect was observed also in single crystals of Crdoped $\mathrm{SrTiO}_{3}$ [42]. This indicates that the effect has a bulk character and is not restricted to the surface. Up to now the physical mechanism responsible for the resistive switching is not known. However, it is clear that the $\mathrm{Cr}$ ion and its different valence states play a crucial role. Electron Paramagnetic Resonance (EPR) has been intensively used to study the electronic character of transition metal ions in $\mathrm{SrTiO}_{3}$ [43]. Therefore, we decided to study Cr-doped $\mathrm{SrTiO}_{3}$ using EPR. In a first step two different $\mathrm{SrTiO}_{3}: \mathrm{Cr}$ single crystals were measured: one with reduced oxygen content and another one as received. The Cr content was 0.2 at. \%. It was found that in the crystals as received the majority of $\mathrm{Cr}$ ions are in the $5+$ valence state. However, a significant amount of $\mathrm{Cr}^{3+}$ is also seen in the EPR spectra. On the other hand, in reduced crystals only $\mathrm{Cr}^{3+}$ centers were observed with no evidence of the presence of $\mathrm{Cr}^{5+}$ ions. These preliminary results show that using EPR it is possible to microscopically characterise different charge states of $\mathrm{Cr}$ in $\mathrm{SrTiO}_{3}$. The next step would be to perform EPR experiments on the samples which show the resistive switching effect. To do this a special sample holder will be developed which allows to apply an electric field on the $\mathrm{SrTiO}_{3}$ crystals directly in the resonance cavity. This will enable to study this interesting effect in situ and possibly clarify its microscopic mechanism.

\subsubsection{Lutetium NQR in $\left(\mathrm{Y}_{1-x} \mathrm{Lu}_{x}\right) \mathrm{Ba}_{2} \mathrm{Cu}_{4} \mathrm{O}_{8}$}

In previous NMR/NQR investigations of $\mathrm{YBa}_{2} \mathrm{Cu}_{4} \mathrm{O}_{8}$ we found that spin-lattice relaxation of $\mathrm{Cu}$ and $\mathrm{O}$ is not only driven by magnetic fluctuations, but also significantly by charge fluctuations. Due to the site symmetry the effect of antiferromagnetic spin fluctuations cancels at the $\mathrm{Y}$ site. In this sense the $\mathrm{Y}$ site represents an ideal location where only the effect of possible charge fluctuations could be detected. Unfortunately the Y nucleus (nuclear spin $I=1 / 2$ ) has no electric quadrupole moment necessary for the detection of charge fluctuations.

One way to overcome this handicap is to replace $\mathrm{Y}$ by another trivalent ion of a rare earth element with a nuclear electric quadrupole moment (nuclear spin $I \geq 1$ ). The coupling of the rare earth nuclear quadrupole moment to the electric field gradient at the nuclear site can then be directly observed in a NQR experiment. In order to avoid disturbing magnetic hyperfine interaction with an on-site electron spin the rare earth element should have a closed outermost shell. Moreover, a large nuclear quadruple moment is of advantage to obtain a strong nuclear quadrupole interaction. The Lutetium isotope ${ }^{175} \mathrm{Lu}$ (nuclear spin $7 / 2$ ) is the best candidate. Like $\mathrm{Y}$ its oxidation state is +3 and it has a large nuclear quadrupole moment $Q=5.68 \cdot 10^{-24} \mathrm{~cm}^{2}$ and a high natural abundance of $97.4 \%$.

In collaboration with S. M. Kazakov and J. Karpinski (Laboratorium für Festkörperphysik, ETH Zürich), who synthesised $\left(\mathrm{Y}_{1-x} \mathrm{Lu}_{x}\right) \mathrm{Ba}_{2} \mathrm{Cu}_{4} \mathrm{O}_{8}$ samples with various compositions $x$, an extensive search for ${ }^{175} \mathrm{Lu}$ NQR was performed in the framework of a diploma thesis [44]. In case of a nuclear spin $7 / 2$ three NQR lines exist. Up to now, in our study of $\left(\mathrm{Y}_{0.8} \mathrm{Lu}_{0.2}\right) \mathrm{Ba}_{2} \mathrm{Cu}_{4} \mathrm{O}_{8}$ and $\left(\mathrm{Y}_{0.7} \mathrm{Lu}_{0.3}\right) \mathrm{Ba}_{2} \mathrm{Cu}_{4} \mathrm{O}_{8}$ performed at $90 \mathrm{~K}$ two of the three Lutetium NQR lines were detected at 39 and $42.5 \mathrm{MHz}$, respectively. Knowledge of these two frequencies allows an estimation of the electric field gradient asymmetry parameter and of the third line's frequency. The third line is expected to be at $3.5 \mathrm{MHz}$ or $17 \mathrm{MHz}$, a frequency range to be covered in the ongoing investigation. 


\section{References}

[1] A.S. Alexandrov and A.M. Bratkovsky, Phys. Rev. Lett. 82, 141 (1999).

[2] A.J. Millis, P.B. Littlewood, and B.I. Shraiman, Phys. Rev. Lett. 74, 5144 (1995).

[3] A. Moreo, S. Yunoki, and E. Dagotto, Science 283, 2034 (1998).

[4] A.S. Alexandrov et al., Phys. Rev. B 64, R140404 (2001).

[5] V.N. Smolyaninova et al., Phys. Rev. B 65, 104419 (2002).

[6] A.S. Alexandrov and N.F. Mott, Int. J. Mod. Phys. 8, 2075 (1994).

[7] K.A. Müller, Physica C 341-348, 11 (2000).

[8] P. Zimmermann et al., Phys. Rev. B 52, 541 (1995).

[9] R. Khasanov et al., cond-mat/0201165 (2002).

[10] J. Hofer et al., Phys. Rev. Lett. 84, 4192 (2000).

[11] G.M. Zhao et al., J. Phys.: Condens. Matter 10, 9055 (1998).

[12] G.M. Zhao et al., Nature (London) 385, 236 (1997).

[13] C. Grimaldi, E. Cappelluti, and L. Pietronero, Europhys. Lett. 42, 667 (1998).

[14] E. Morenzoni et al., J. Appl. Phys. 81, 3340 (1997).

[15] T.J. Jackson et al., Phys. Rev. Lett. 84, 4958 (2000).

[16] Proc. Int. Workshop on Anharmonic Properties of High- $T_{c}$ Cuprates edited by D. Mihailovic, G. Ruani, E. Kaldis, and K. A. Müller) 118-146 (World Scientific, Singapore, 1994).

[17] A. Shengelaya et al., Phys. Rev. Lett. 83, 5142 (1999).

[18] D.W. Cooke et al., Phys. Rev. B 41, 4801 (1990).

[19] J. Nagamatsu et al., Nature 410, 63 (2001).

[20] Eva Pavarini and I.I. Mazin, Phys. Rev. B 64, 140504 (2001).

[21] A.V. Tsvyashchenko et al., Solid State Commun. 119, 153 (2001).

[22] M. Mali, J. Roos, A. Shengelaya, and H. Keller, Phys. Rev. 65, 100518(R) (2002).

[23] M. Angst et al., cond-mat/0112166 (Phys. Rev. Lett., in press).

[24] G. Ravikumar et al., Phys. Rev. B 63, 024505 (2000).

[25] M.R. Eskildsen et al., Phys. Rev. Lett. 86, 5148 (2001).

[26] V.G. Kogan et al., Phys. Rev. B 55, R8693 (1997).

[27] M. Willemin et al., Phys. Rev. B 58, R5940 (1998).

[28] N. Avraham et al., Nature 411, 451 (2001). 
[29] A. Schilling et al., Phys. Rev. B 58, 11157 (1998).

[30] M. Willemin et al., Phys. Rev. Lett. 81, 4236 (1998).

[31] A. Schilling et al., Phys. Rev. B 61, 3592 (2000).

[32] A. Schilling et al., Phys. Rev. B 65, 054505 (2002).

[33] C.M. Aegerter et al., Phys. Rev. B 57, 14511 (1998).

[34] S.L. Lee et al., Phys. Rev. Lett. 71, 862 (1993).

[35] M. Corti et al., Phys. Rev. B 54, 9469 (1996).

[36] F. Bouquet et al., Nature 411, 448 (2001).

[37] A. Schilling et al., Phys. Rev. Lett. 76, 4833 (1997).

[38] P.G. de Gennes, Superconductivity of metals and alloys, (Addison-Wesley, Massachusetts, 1989).

[39] P. Zimmermann et al., Hyperfine Interactions 63, 33 (1990).

[40] J.M. Delrieu, J. Phys. F: Metal Phys. 3, 893 (1973).

[41] A. Beck et al., Appl. Phys. Lett. 77, 139 (2000).

[42] Y. Watanabe et al., Appl. Phys. Lett. 78, 3738 (2001).

[43] K.A. Müller et al., Solid State Commun. 85, 381 (1993).

[44] Philipp Schneider, Diploma thesis, Physik-Institut, Universität Zürich, 2001. 